\title{
Paliperidone extended-release tablets in Chinese patients with schizophrenia: meta-analysis of randomized controlled trials
}

This article was published in the following Dove Press journal:

Neuropsychiatric Disease and Treatment

23 July 2015

Number of times this article has been viewed

\author{
Shangli Cai ${ }^{1,2}$ \\ Huafei Lu \\ Zhihua $\mathrm{Bai}^{2}$ \\ Renrong Wu' \\ Jingping Zhao'
}

'Mental Health Institute of the Second Xiangya Hospital, National Technology Institute of Psychiatry, Key Laboratory of Psychiatry and Mental Health of Hunan Province, Central South University, Hunan, ${ }^{2} J a n s s e n$ Research and Development, Beijing, People's Republic of China
Correspondence: Jingping Zhao Mental Health Institute of the Second Xiangya Hospital, National Technology Institute of Psychiatry, Key Laboratory of Psychiatry and Mental Health of Hunan Province, Central South University, I39 Middle Renmin Road, Changsha, Hunan 4I00II, People's Republic of China.

Tel +8673185554052

Fax +86 73। 85554052

Email jpzhao@outlook.com
Background: Previous meta-analyses have compared paliperidone extended-release (ER) tablets with other antipsychotics, but none have involved Chinese patients or studies from People's Republic of China. Further, the results of these meta-analyses may not be applicable to Chinese patients. In the present study, we evaluated the efficacy, safety, and acceptability of paliperidone ER compared with other second-generation antipsychotics (SGAs) for Chinese patients with schizophrenia.

Methods: Randomized controlled studies of paliperidone ER and other SGAs as oral monotherapy in the acute phase treatment of schizophrenia were retrieved from Medline, Embase, and the Cochrane Library (CENTRAL), as well as from Chinese databases including the China National Knowledge Infrastructure, Wanfang, and VIP Information/Chinese Scientific Journals Database. We pooled data on response rates, chance of withdrawal due to adverse events, probability of adverse events, and odds of withdrawal for any reason.

Results: Fifty randomized controlled trials were identified. The response rate for paliperidone ER was significantly higher than that of other pooled SGAs (risk ratio [RR] 0.83, 95\% confidence interval [CI] 0.72-0.96) and ziprasidone (RR 0.57, 95\% CI 0.39-0.82). Paliperidone ER significantly reduced the chance of withdrawal due to adverse events and the chance of any adverse events compared with other pooled SGAs (RR 0.32, 95\% CI $0.17-0.58$ and RR $0.88,95 \%$ CI $0.79-0.97$ ) and risperidone (RR $0.31,95 \%$ CI $0.14-0.67$ and RR $0.70,95 \%$ CI 0.57-0.86). The incidence of extrapyramidal symptoms on paliperidone ER was comparable with other pooled SGAs (RR 0.94, 95\% CI 0.66-1.35) and significantly lower than that of risperidone (RR 0.56, 0.41-0.77) but higher than that of olanzapine (RR 1.88, 95\% CI 1.05-3.36). Paliperidone ER was superior to other pooled SGAs (RR $0.32,95 \%$ CI $0.21-0.49$ and RR $0.50,95 \%$ CI $0.35-0.72$ ) and olanzapine (RR $0.23,95 \%$ CI $0.15-0.33$ and RR $0.33,95 \%$ CI $0.23-0.47$ ) as far as weight gain and somnolence were concerned. Further, prolactin-related adverse events caused by paliperidone ER were comparable with other pooled SGAs (RR 1.30, 95\% CI 0.73-2.33), but outnumbered those caused by olanzapine (RR 7.53, 95\% CI 2.05-27.71).

Conclusion: Paliperidone ER is efficacious, safe, and well accepted when compared with other pooled SGAs for the treatment of Chinese patients with schizophrenia.

Keywords: paliperidone extended-release, second-generation antipsychotics, schizophrenia, meta-analysis

\section{Introduction}

Schizophrenia is a severe and chronic psychotic disorder with a significant social and economic impact. The lifetime prevalence of schizophrenia is $0.30 \%-0.66 \%$ worldwide, ${ }^{1}$ and about 781/100,000 in People's Republic of China, indicating that there are more than ten million schizophrenia patients in this country. ${ }^{2}$ Antipsychotic drugs 
are a key component of treatment for schizophrenia. Several commonly prescribed second-generation antipsychotics (SGAs) in People's Republic of China include risperidone, clozapine, olanzapine, aripiprazole, and quetiapine. ${ }^{3}$

Paliperidone extended-release (ER; Invega ${ }^{\mathrm{TM}}$, Johnson \& Johnson Pharmaceuticals, Titusville, NJ, USA) is an SGA with unique pharmacological/pharmacokinetic characteristics and an innovative drug delivery system, and is newly introduced in People's Republic of China. Paliperidone is the active metabolite of risperidone, ie, 9-hydroxyrisperidone, which has high affinity for serotonin $2 \mathrm{~A}\left(5-\mathrm{HT}_{2 \mathrm{~A}}\right)$ and dopamine $\mathrm{D}_{2}$ receptors and are two targets for psychosis treatment. ${ }^{4}$ It is also an antagonist of the histamine $H_{1}$ receptor and the $\alpha_{1}$ and $\alpha_{2}$ adrenoceptors. Paliperidone has an affinity for $5-\mathrm{HT}_{7}$, dopamine $\mathrm{D}_{3}$, and $\alpha_{2 \mathrm{~A}}$ adrenoceptors receptors, indicating a role as an antidepressant and improving sleep and cognition. ${ }^{5-7}$ Paliperidone ER is formulated within an osmotically controlled-release oral delivery system $\left(\right.$ OROS $^{\circledR}$; ALZA Corporation, Mountain View, CA, USA) that minimizes peak-trough fluctuations and does not require dose titration. ${ }^{8}$ Paliperidone ER is less prone to hepatic drug-drug interactions due to the lack of cytochrome P450 inhibition and less hepatic metabolism. ${ }^{9}$ As a result, there is only a small reduction in total exposure to paliperidone ER in patients with hepatic impairment. ${ }^{10}$

A number of studies have investigated the efficacy, safety, and acceptability of paliperidone ER in patients with schizophrenia. ${ }^{11-14}$ Previous meta-analyses have compared paliperidone ER with other oral SGAs, but none have involved Chinese patients or studies from People's Republic of China. ${ }^{15,16}$ Further, the results of these meta-analyses may not be applicable to Chinese patients. Studies have shown that several single nucleotide polymorphisms are overexpressed in Caucasian patients with schizophrenia but not in their Chinese counterparts, and vice versa. ${ }^{17,18}$ Also, data from Phase III trials have demonstrated that the average weight of Chinese study participants was much less than that of Western participants. ${ }^{12,19}$ In addition, the incidence of paliperidone ER-related extrapyramidal side effects (EPS) in Chinese patients experiencing an acute exacerbation of schizophrenia has been reported to be around $40 \%,{ }^{19,20}$ which is much higher than in patients from other countries (4\%-8\%). ${ }^{11-13}$ Despite mounting evidence that patients in People's Republic of China are different, no meta-analysis comparing paliperidone ER with other SGAs in Chinese patients with schizophrenia has ever been published.

This meta-analysis specifically aimed to compare the efficacy, safety, and acceptability of paliperidone ER with that of other SGAs in Chinese patients with schizophrenia in the hope of providing reference information for future studies and clinical application.

\section{Materials and methods}

\section{Search strategy}

Databases including Medline, PubMed, Embase, and the Cochrane Library (CENTRAL) were searched to identify eligible studies in English, while databases including the People's Republic of China National Knowledge Infrastructure, Wan Fang, and the VIP Information/Chinese Scientific Journals Database were searched for studies published in Chinese to October 20, 2014.

Keywords for the literature search were associated with populations and treatment. Keywords for populations included "China", "Chinese", "Hong Kong", "Taiwan", and "Taiwanese", those for treatment included "paliperidone extended-release", "paliperidone ER", "9-OH risperidone", and "INVEGA", and the disease name was "schizophrenia". Keywords for each category were connected using the Boolean operator AND. The literature search was carried out by two authors (HFL and ZHB) to ensure accuracy.

\section{Inclusion and exclusion criteria}

Inclusion criteria were: all patients included had to be Chinese patients with schizophrenia living in mainland China, Taiwan, or Hong Kong; the dose and duration of treatment had to be clearly stated; the studies included should be randomized controlled trials (RCTs); all patients involved were in the acute phase of schizophrenia and treated with oral SGA as monotherapy; the treatment duration of each study had to be 4-12 weeks; and the Chinese literature had to be published in Peking University or Chinese core journals. Exclusion criteria were: duplicate publication; lack of quantitative data on efficacy, adverse events, or withdrawal for any reason; and economic evaluations and study protocols.

\section{Assessment of study quality and data extraction}

The methodological quality of the included studies was assessed with the Jadad scale, used to assess randomization, double-blind, and dropouts. ${ }^{21}$ The primary outcome was the response rate, while secondary outcomes included: odds of withdrawal due to adverse events; probability of any adverse events; probability of EPS, weight gain, somnolence, and prolactin-related adverse events; and odds of withdrawal for any reason. Subgroup analyses based on treatment arm and Jadad score were also performed for each outcome.

\section{Statistical analysis}

The meta-analysis was performed using Review Manager (Revman) version 5.3, provided by the Cochrane Collaborative (http:// www.cc-ims.net/RevMan). Risk ratio (RR) was estimated along 
with the $95 \%$ confidence interval (CI). Study heterogeneity was measured using the $\chi^{2}$ test and $I^{2}$ statistics. A $\chi^{2}$ value of $P<0.05$ and an $I^{2}$ of $\geq 50 \%$ indicated heterogeneity. In this case, a random effect model and the Dersimonian-Laird method would be performed. When the $\chi^{2}$ value was $P>0.05$ and $I^{2}$ was $<50 \%$, a fixed-effect model was used and the Mantel-Haenszel method was performed. Finally, funnel plots were inspected visually to explore the possibility of publication bias.

\section{Results}

\section{Literature search}

In total, 97 English studies were retrieved from PubMed, Medline, Embase, and the Cochrane Library, while 818 Chinese studies were retrieved from People's Republic of China National Knowledge Infrastructure, Wan Fang, and VIP Information/Chinese Scientific Journals Database. Of these, 246 Chinese studies were published in Peking University or Chinese core journals. After duplicates were removed, 188 records were considered valid. A further 128 articles were excluded after initial screening of titles and abstracts, and another ten studies were excluded for lack of relevant data, not including a treatment duration of 4-12 weeks, or not being an RCT. Finally, a total of 50 RCTs were retrieved (Supplementary Materials, Figure 1 and Table S1), among which 38 were included in the efficacy analysis set (Table S2), 44 in the adverse events analysis set (Table S3), and 28 in the analysis set for withdrawal for any reason (Table S4). In these 50 RCTs, paliperidone ER was compared with risperidone, olanzapine, aripiprazole, ziprasidone, quetiapine, or clozapine as monotherapy in $17,20,4,7,1$, and 1 studies, respectively. Numbers of patients treated with paliperidone, risperidone, olanzapine, aripiprazole, ziprasidone, quetiapine, or clozapine was $2,054,681,858,126,317,53$, and 25 , respectively. One study was published in an international peer-reviewed journal, 24 were in Peking University core journals, and 25 in Chinese core journals. Five RCTs were double-blind, 15 were openlabel, and 30 did not report if they were open-label, singleblind, or double-blind. Twenty-four trials were classified as being of high quality (Jadad score $\geq 3$ ) and 26 trials were of low quality (Jadad score of $<3$ ). The characteristics of these 50 studies are shown in Table S1.

\section{Efficacy outcomes}

The primary outcome was efficacy, as measured by the response rate defined in each study. Of the $38 \mathrm{RCTs}$ in the efficacy analysis set (Table S2), most (25/38) defined treatment response as an at least $25 \%$ reduction in Positive and Negative Syndrome Scale (PANSS) total score (30\% in a few studies). In these $38 \mathrm{RCTs}, 1,324$ of

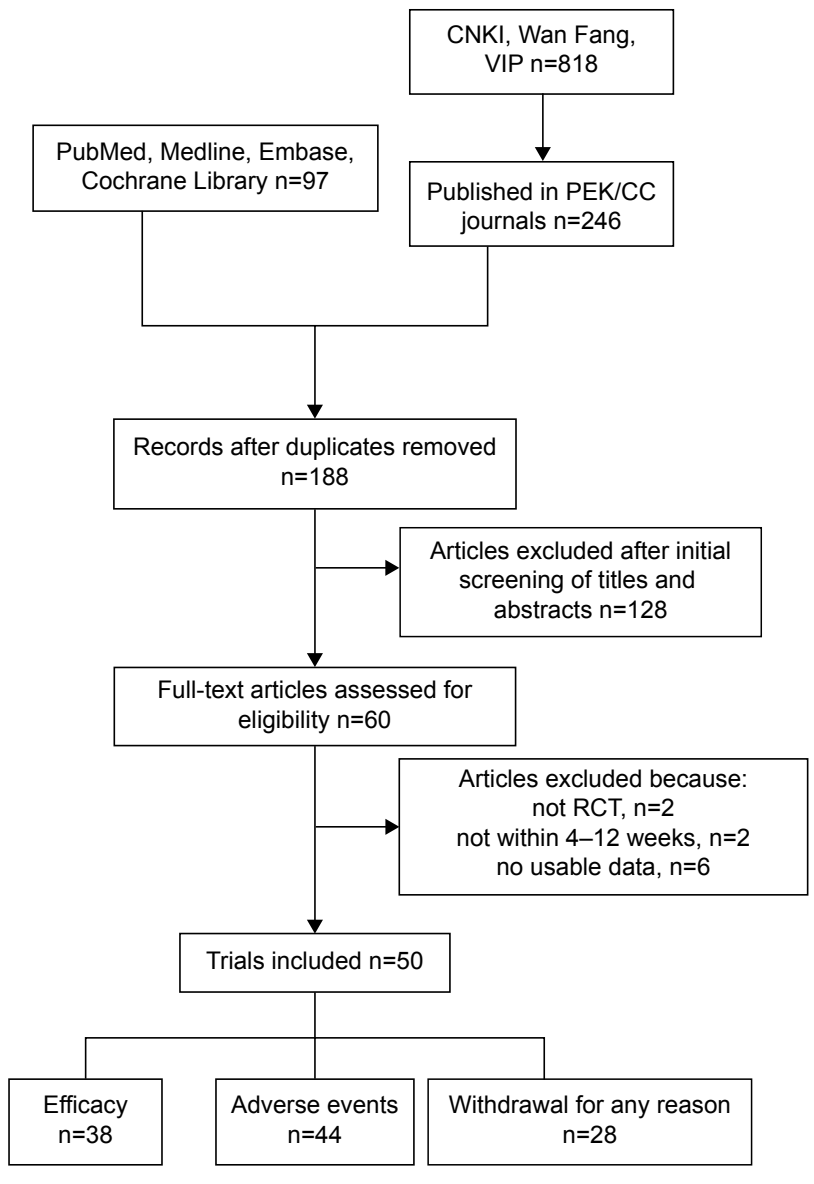

Figure I Flow diagram of literature search and selection.

Abbreviations: RCT, randomized controlled trial; CNKI, China National Knowledge Infrastructure; PEK, Peking University core journals; CC, Chinese core journals; VIP, VIP Information/Chinese Scientific Journals Databases.

1,570 patients treated with paliperidone ER responded, while 1,281 of 1,578 patients treated with other SGAs as monotherapy responded. No significant heterogeneity was found between the 38 RCTs $\left(I^{2}=0 \%, P=0.99\right)$. Paliperidone ER was significantly more efficacious than other pooled SGAs (RR 0.83, 95\% CI 0.72-0.96, $P=0.01$; Figure 2A). Subgroup analysis demonstrated that the efficacy of paliperidone ER was not inferior to that of risperidone (RR 0.88, 95\% CI 0.65-1.19, $P=0.40$ ), olanzapine (RR $0.95,95 \%$ CI $0.77-1.18, P=0.66$ ), or aripiprazole (RR 0.53, 95\% CI 0.24-1.19, $P=0.12$ ), and was significantly better than that of ziprasidone (RR 0.57, 95\% CI 0.39-0.82, $P=0.003$; Figure 2A). Funnel plots showed slightly asymmetric scattered dots, suggesting the possibility of publication bias (Figure 2B). In addition, the response rate of paliperidone ER was higher than that of other pooled SGAs in both the high Jadad score subgroup (RR 0.85, 95\% CI $0.69-1.04, P=0.12$ ) and the low Jadad score subgroup (RR $0.82,95 \%$ CI $0.66-1.01, P=0.06)$. However, the difference was not statistically significant, probably due to the limited number of trials in each subgroup (Figure 2C). 


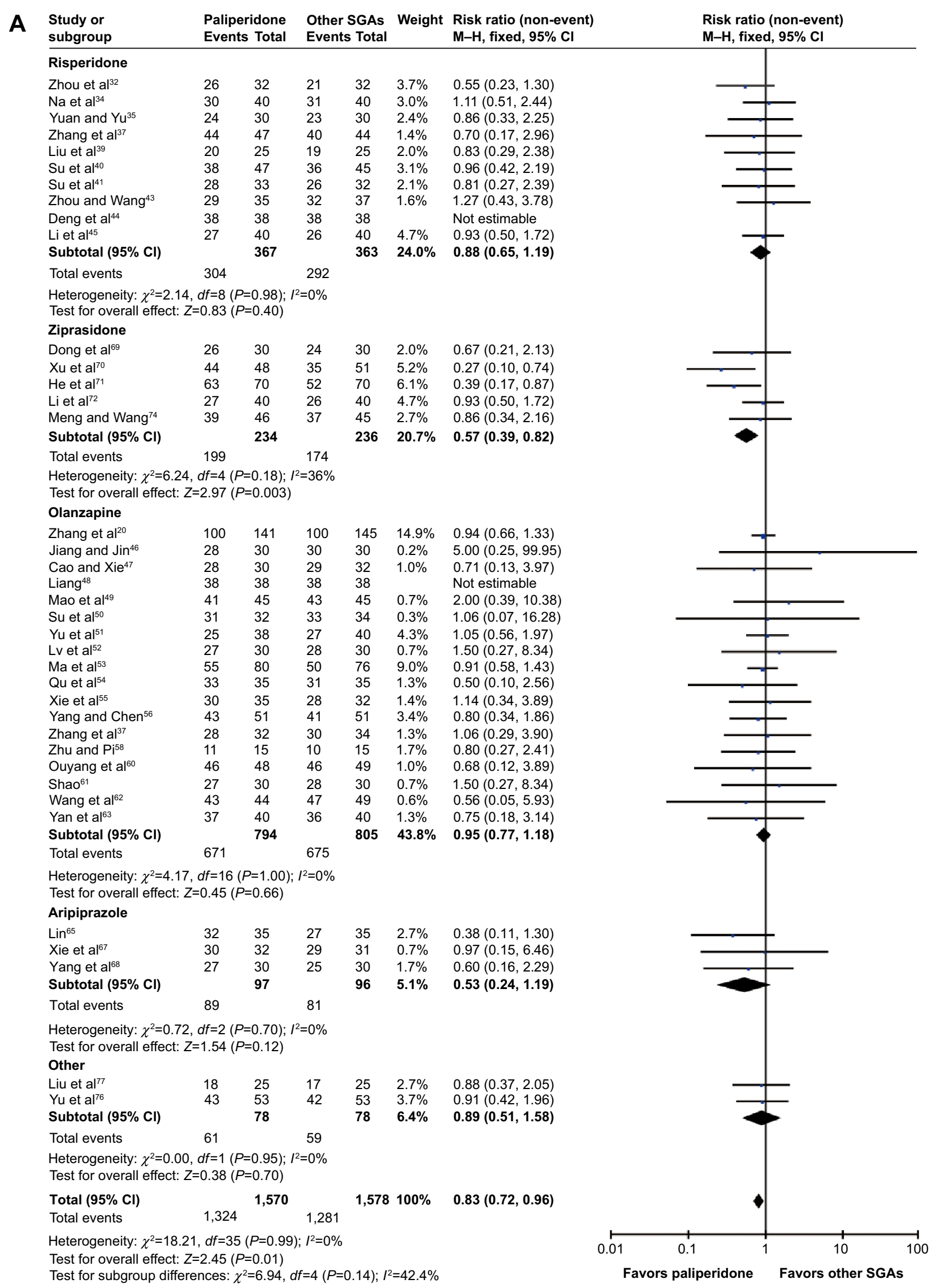

Figure 2 (Continued) 
B

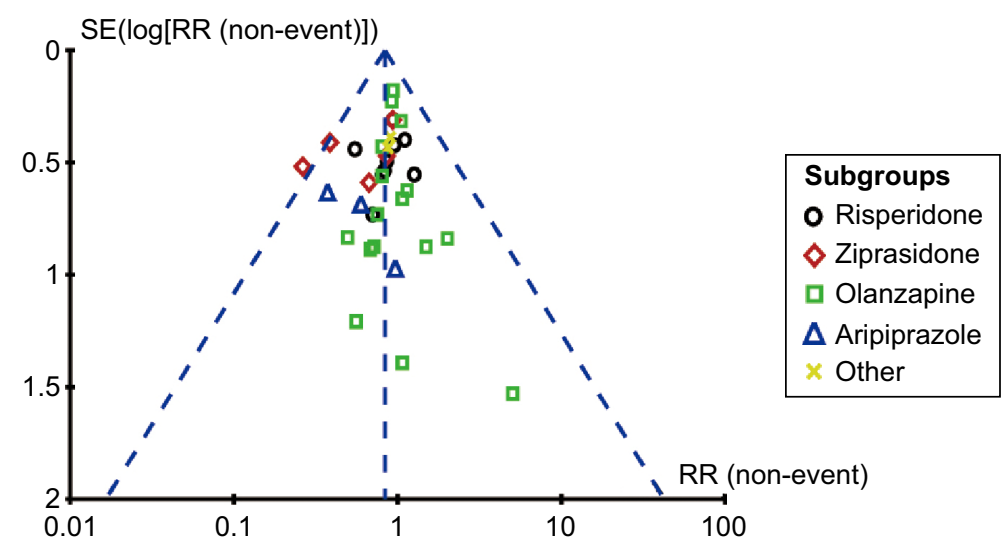

\section{C}

\begin{tabular}{|c|c|c|c|c|c|c|}
\hline $\begin{array}{l}\text { Study or } \\
\text { subgroup }\end{array}$ & $\begin{array}{l}\text { Paliper } \\
\text { Events }\end{array}$ & $\begin{array}{l}\text { idone } \\
\text { Total }\end{array}$ & $\begin{array}{l}\text { Other S } \\
\text { Events }\end{array}$ & $\begin{array}{l}\text { GAs } \\
\text { Total }\end{array}$ & Weight & $\begin{array}{l}\text { Risk ratio (non-e } \\
\text { M-H, fixed, } 95 \%\end{array}$ \\
\hline \multicolumn{7}{|c|}{ Jadad score $(3,4$, and 5$)$} \\
\hline Xie et al ${ }^{67}$ & 30 & & 29 & 31 & $0.7 \%$ & $0.97(0.15,6.46)$ \\
\hline Zhang et $\mathrm{al}^{20}$ & 100 & 141 & 100 & 145 & $14.9 \%$ & $0.94(0.66,1.33)$ \\
\hline Jiang and $\mathrm{Jin}^{46}$ & 28 & 30 & 30 & 30 & $0.2 \%$ & $5.00(0.25,99.95$ \\
\hline Liang $^{48}$ & 38 & 38 & 38 & 38 & & Not estimable \\
\hline $\mathrm{Xi}$ & 30 & 35 & 28 & 32 & $1.4 \%$ & $1.14(0.34,3.89)$ \\
\hline Ouyang et $a^{160}$ & 46 & 48 & 46 & 49 & $1.0 \%$ & $0.68(0.12,3.89)$ \\
\hline & 37 & & 36 & 40 & $1.3 \%$ & $18,3.14)$ \\
\hline Zhou et al ${ }^{32}$ & 26 & 32 & 21 & 32 & $3.7 \%$ & $0.55(0.23,1.30)$ \\
\hline $\mathrm{Na}$ et $\mathrm{a}^{34}$ & 30 & 40 & 31 & 40 & $3.0 \%$ & $1.11(0.51,2.44)$ \\
\hline & 44 & 47 & 40 & 44 & & \\
\hline Su et al & 38 & 47 & 36 & 45 & $3.1 \%$ & $0.96(0.42,2.19)$ \\
\hline Su et al & 28 & 33 & 26 & 32 & $2.1 \%$ & $0.81(0.27,2.39)$ \\
\hline Deng & 38 & 38 & 38 & 38 & & Not estimable \\
\hline & 27 & 40 & 26 & 40 & $4.7 \%$ & $0.93(0.50,1.72)$ \\
\hline He et $a^{71}$ & 63 & 70 & 52 & 70 & $6.1 \%$ & $0.39(0.17,0.87)$ \\
\hline \multirow{3}{*}{$\begin{array}{l}\text { Li et al }{ }^{27} \\
\text { Subtolal }(95 \% \mathrm{Cl}) \\
\text { Total events }\end{array}$} & 27 & & 26 & & $4.7 \%$ & $0.93(0.50,1.72)$ \\
\hline & & 751 & & 746 & $48.3 \%$ & $0.85(0.69,1.04)$ \\
\hline & 630 & & 603 & & & \\
\hline
\end{tabular}

Heterogeneity: $\chi^{2}=7.35, d f=13(P=0.88) ; P^{2}=0 \%$

Test for overall effect: $Z=1.55(P=0.12)$

Jadad score (1 and 2)

$\operatorname{Lin}^{65}$

Yang et al $^{68}$

Liu et al $^{77}$

$\mathrm{Cao}$ and $\mathrm{Xie}^{47}$

Mao et $\mathrm{al}^{49}$

Su et $\mathrm{al}^{50}$

Yu et $\mathrm{al}^{51}$

$\mathrm{LV}^{52}$

Ma et al ${ }^{53}$

Qu et $a^{54}$

Yang and Chen 33

Zhang and $\mathrm{Pei}^{57}$

Zhu and $\mathrm{Pi}^{58}$

Shao ${ }^{61}$

Wang et $\mathrm{al}^{62}$

Yu et $\mathrm{al}^{76}$

Yuan and $\mathrm{Yu}^{35}$

Liu et al ${ }^{39}$

Zhou and Wang ${ }^{43} \quad 20$

Dong et al ${ }^{69} \quad 26$

Xu et $\mathrm{al}^{70}$

Meng and Wang ${ }^{74} \quad 39$

Subtotal $(95 \% \mathrm{Cl})$

Total events

32
27

$\begin{array}{ll}35 & 27 \\ 30 & 25 \\ 25 & 17\end{array}$

$\begin{array}{ll}27 & 35 \\ 25 & 30\end{array}$

$35 \quad 2.7 \%$

$1.7 \%$
$2.7 \%$

$0.38(0.11,1.30)$

$0.60(0.16,2.29)$

$0.88(0.37,2.05)$

$1.0 \%$

$0.71(0.13,3.97)$

$0.7 \%$

$2.00(0.39,10.38)$

$1.06(0.07,16.28)$

$1.05(0.56,1.97)$

$1.50(0.27,8.34)$

$0.91(0.58,1.43)$

$0.50(0.10,2.56)$

$0.80(0.34,1.86)$

$1.06(0.29,3.90)$

$0.80(0.27,2.41)$

$1.50(0.27,8.34)$

$0.56(0.05,5.93)$

$0.91(0.42,1.96)$

$0.86(0.33,2.25)$

$0.83(0.29,2.38)$

$1.27(0.43,3.78)$

$0.67(0.21,2.13)$

$0.27(0.10,0.74)$

$0.86(0.34,2.16)$

694

$0.82(0.66,1.01)$

Heterogeneity: $\chi^{2}=10.86, d f=21(P=0.97) ; P^{2}=0 \%$

Test for overall effect: $Z=1.90(P=0.06)$

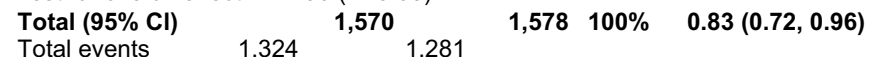

Heterogeneity: $\chi^{2}=18.21, d f=35(P=0.99) ; P^{2}=0 \%$

Test for overall effect: $Z=2.45(P=0.01)$

Test for subgroup differences: $\chi^{2}=0.06, d f=1(P=0.81) ; P^{2}=0 \%$

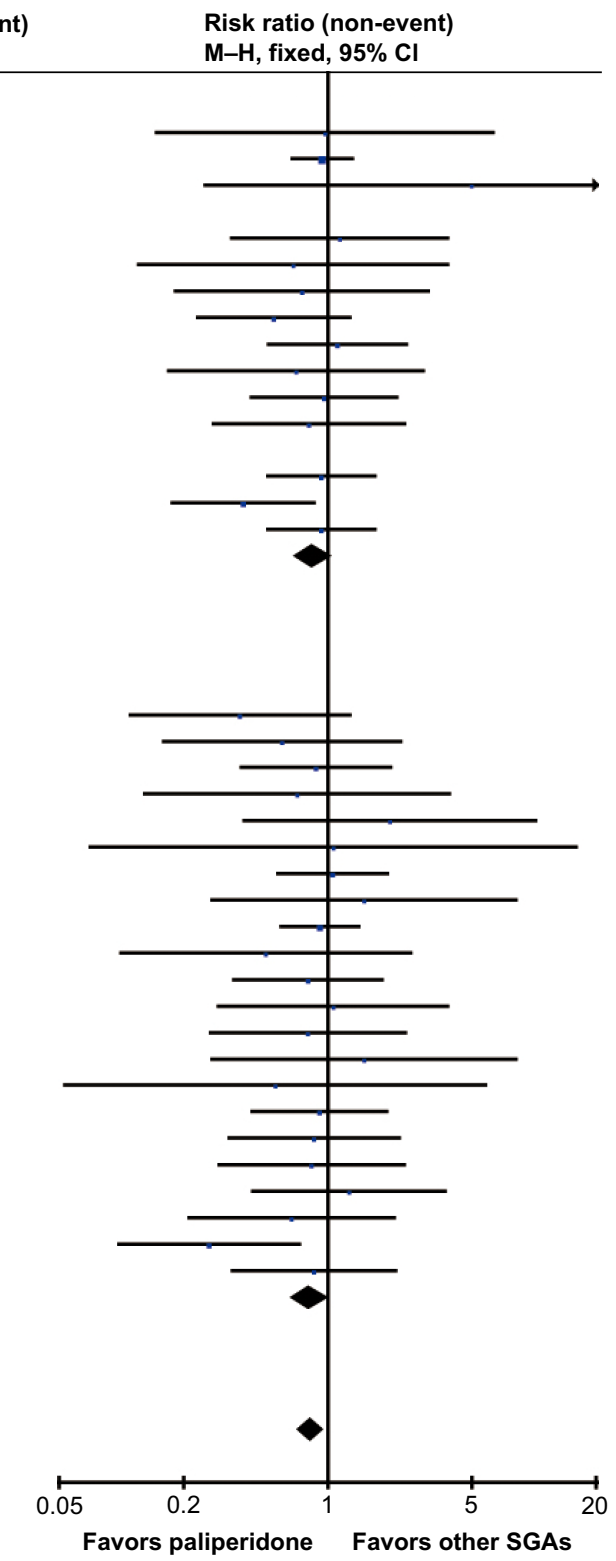

Figure 2 Response rates.

Notes: Subgroup analysis was carried out based on treatment arms (A) and Jadad scores (C). Funnel plots were used to evaluate potential publication bias (B). RR is represented by blue dots was estimated along with the $95 \%$ confidence interval $(\mathrm{Cl})$ represented by horizontal lines.

Abbreviations: $\mathrm{Cl}$, confidence interval; $\mathrm{M}-\mathrm{H}$, Mantel-Haenszel method; RR, risk ratio; SGAs, second-generation antipsychotics; SE, standard error. 
Overall, our results suggest that the efficacy of paliperidone ER is better than that of other pooled SGAs, comparable with that of risperidone, olanzapine, and aripiprazole, and superior to ziprasidone.

\section{Safety outcomes}

Of the 50 RCTs included, 44 reported data on adverse events (Table S3). Adverse events related to the treatment of paliperidone ER included EPS (akathisia, acute dystonia, Parkinsonism), somnolence, weight gain, and prolactin-related adverse events, of which EPS is the most common.

\section{Withdrawal due to adverse events and total adverse events}

Eighteen studies reported odds of withdrawal due to adverse events. Paliperidone ER had significantly lower odds of withdrawal due to adverse events than the other pooled SGAs (RR $0.32,95 \%$ CI $0.17-0.58, P=0.0002$; Figure $3 \mathrm{~A}$ ). In addition, the odds of withdrawal due to adverse events in the paliperidone ER group were significantly lower than in the risperidone group ( $\mathrm{RR} 0.31,95 \% \mathrm{CI} 0.14-0.67, P=0.003$ ) and comparable with those of olanzapine (RR 0.33, 95\% CI $0.11-0.99, P=0.05$ ), ziprasidone (RR 0.33, 95\% CI 0.01-7.95, $P=0.50$ ), and aripiprazole (RR 0.33, 95\% CI 0.01-7.91, $P=0.50$; Figure $3 \mathrm{~A})$. Eighteen RCTs reported data on the probability of adverse events. Our meta-analysis results indicated that paliperidone ER produced significantly fewer adverse events than other pooled SGAs (RR 0.88, 95\% CI 0.79-0.97, $P=0.01)$. Further, the chance of any adverse events in the paliperidone ER group was significantly less than that in the risperidone group (RR $0.70,95 \%$ CI $0.57-0.86, P=0.0008$ ) and comparable with that in the olanzapine group (RR 1.00, 95\% CI 0.86-1.15, $P=0.95$; Figure 3B) and ziprasidone group (RR 0.96, 95\% CI 0.72-1.28, $P=0.80$; Figure 3B).

\section{Extrapyramidal symptoms}

EPS are the most common adverse events associated with antipsychotic drugs. Taking akathisia as an example, our meta-analysis demonstrated that paliperidone ER was not different from the other pooled SGAs (RR 0.94, 95\% CI 0.66-1.35, $P=0.74$; Figure 4A). Further, paliperidone ER caused significantly less akathisia than risperidone (RR 0.56 , 95\% CI $0.41-0.77, P=0.0003$ ), but was more likely than olanzapine to induce akathisia (RR 1.88, 95\% CI 1.05-3.36, $P=0.03$; Figure 4A).

\section{Body weight gain and somnolence}

Body weight gain and sedation are adverse events commonly associated with antipsychotic drugs. Of the 44 studies enrolled in the adverse events analysis set, 20 reported the number of patients with weight gain $\geq 7 \%$ and 30 reported the number of patients with somnolence. Paliperidone ER caused significantly less weight gain than the other pooled SGAs (RR 0.32, 95\% CI 0.21-0.49, $P<0.00001$ ) as well as, specifically olanzapine (RR $0.23,95 \%$ CI $0.15-0.33$, $P<0.00001)$. Paliperidone ER was better than risperidone (RR 0.51, 95\% CI 0.26-1.03, $P=0.06$ ), but not significantly so (Figure 4B). Patients treated with paliperidone ER were much less likely to develop somnolence than those treated with other pooled SGAs (RR 0.50, 95\% CI 0.35-0.72, $P=0.0002)$ or olanzapine specifically (RR $0.33,95 \% \mathrm{CI}$ 0.23-0.47, $P<0.00001)$. Paliperidone ER was comparable with risperidone (RR 1.08, 95\% CI 0.63-1.87, $P=0.78$ ) and ziprasidone (RR 1.35, 95\% CI 0.13-13.58, $P=0.80$ ) with regard to somnolence (Figure $4 \mathrm{C}$ ). These results suggest that paliperidone ER has a relatively mild impact on weight gain and somnolence.

\section{Prolactin-related adverse events}

Irregular menstruation was one of the most common prolactinrelated adverse events. Ten studies reported numbers of patients with irregular menstruation. Our meta-analysis results demonstrated that paliperidone ER was not inferior to other pooled SGAs with regard to the impact on menstruation (RR 1.30, 95\% CI 0.73-2.33, P=0.37). Paliperidone ER was also better than risperidone (RR 0.38, 95\% CI 0.12-1.21, $P=0.10$, not statistically significant), but significantly more likely than olanzapine to cause irregular menstruation (RR 7.53, 95\% CI 2.05-27.71, $P=0.002$; Figure 4D).

Our results suggest that the incidence of EPS caused by paliperidone ER is comparable with other pooled SGAs but significantly lower than that caused by risperidone and higher than that caused by olanzapine. Paliperidone ER is superior to other pooled SGAs and olanzapine as far as weight gain and somnolence are concerned. Further, prolactin-related adverse events caused by paliperidone ER are comparable with other pooled SGAs, but outnumber those caused by olanzapine.

\section{Withdrawal for any reason}

Withdrawal for any reason was used as a measure of acceptability. Twenty-eight of the 50 studies included in this meta-analysis reported odds of withdrawal for any reason (Table S4). Paliperidone ER was significantly better than other pooled SGAs (RR 0.73, 95\% CI 0.55-0.97, $P=0.03$; Figure 5A). Visual inspection of funnel plots for the odds of withdrawal for any reason did not suggest any publication bias (Figure 5B). In addition, paliperidone ER had a significantly 


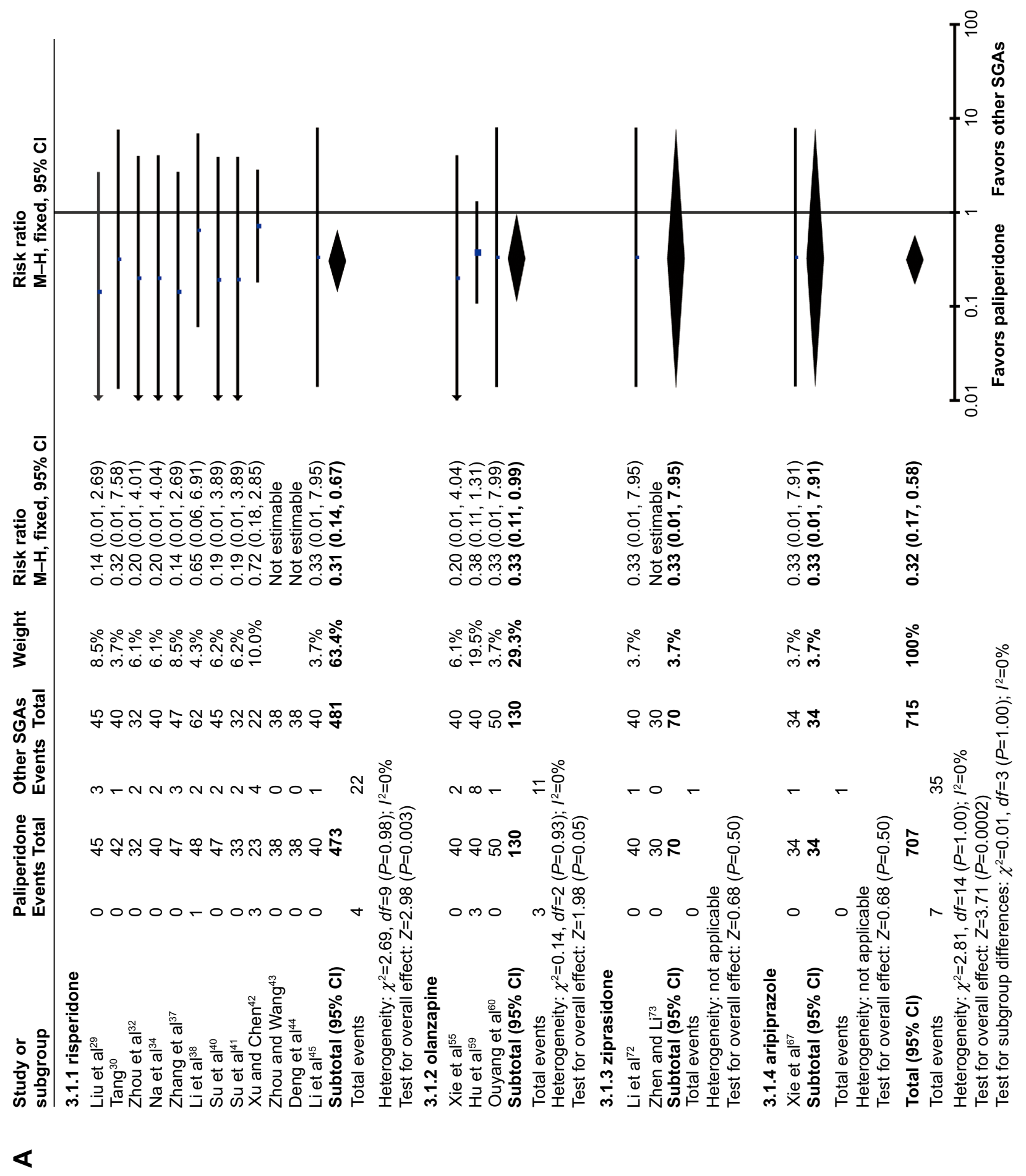




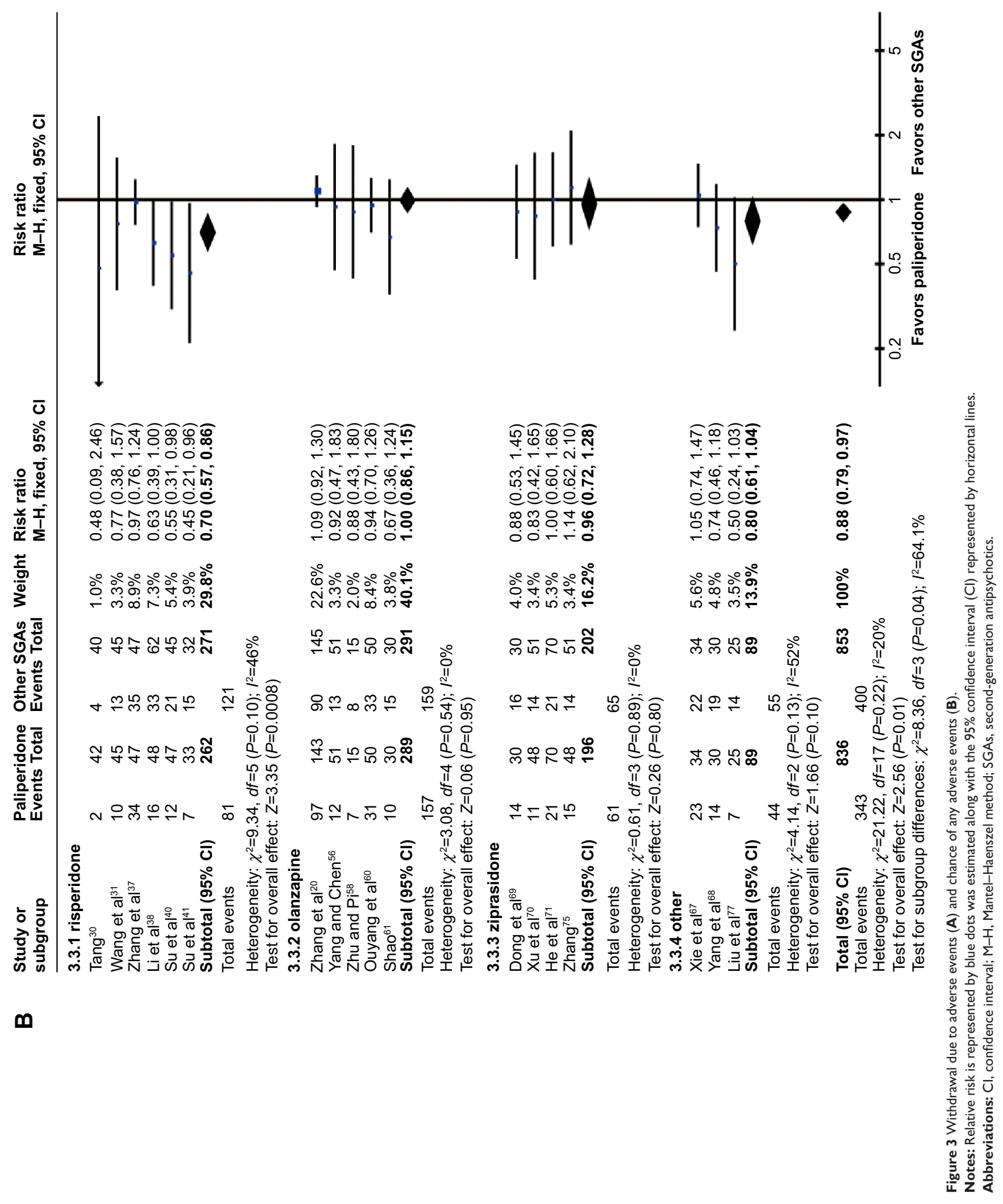




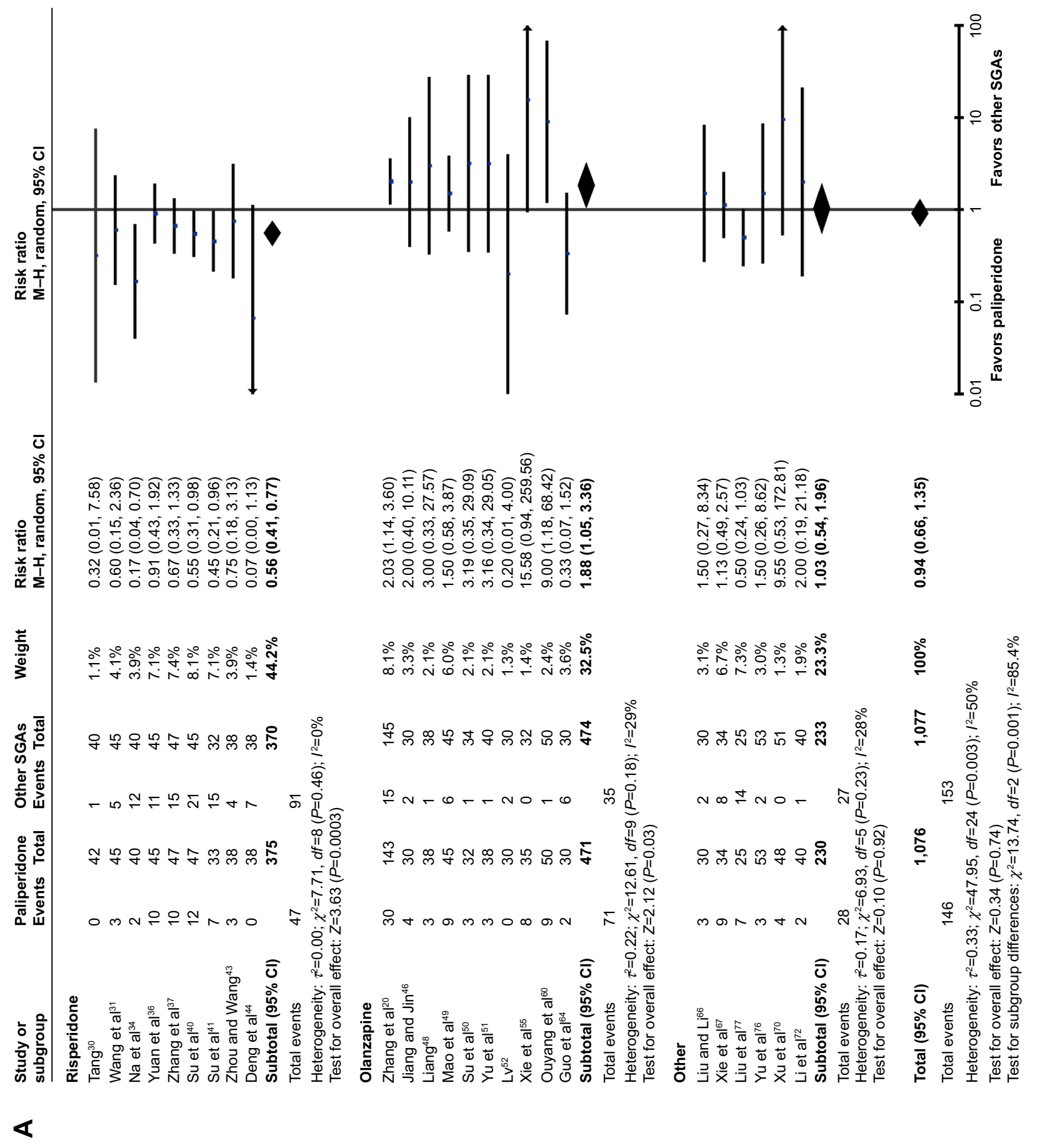




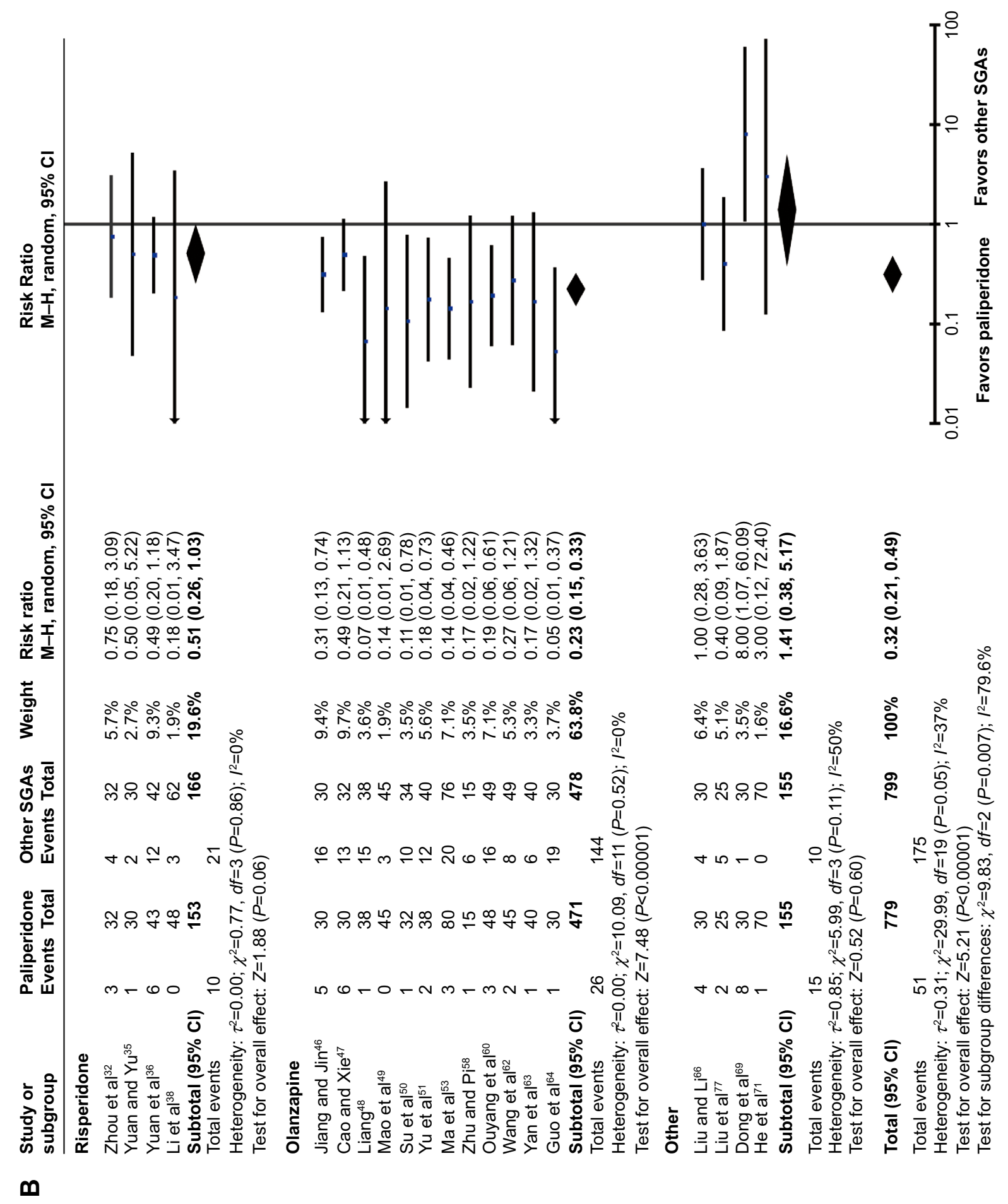




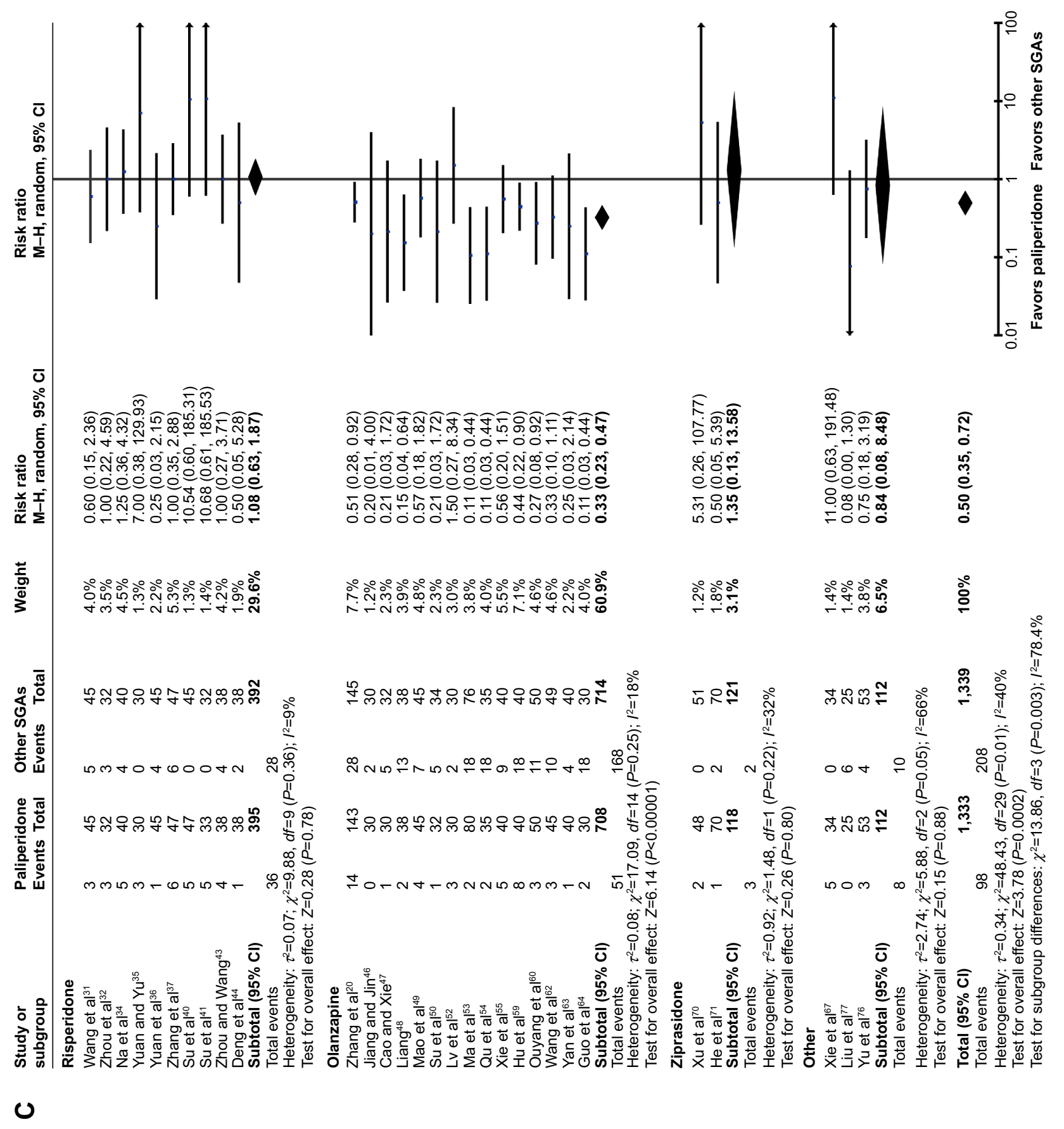




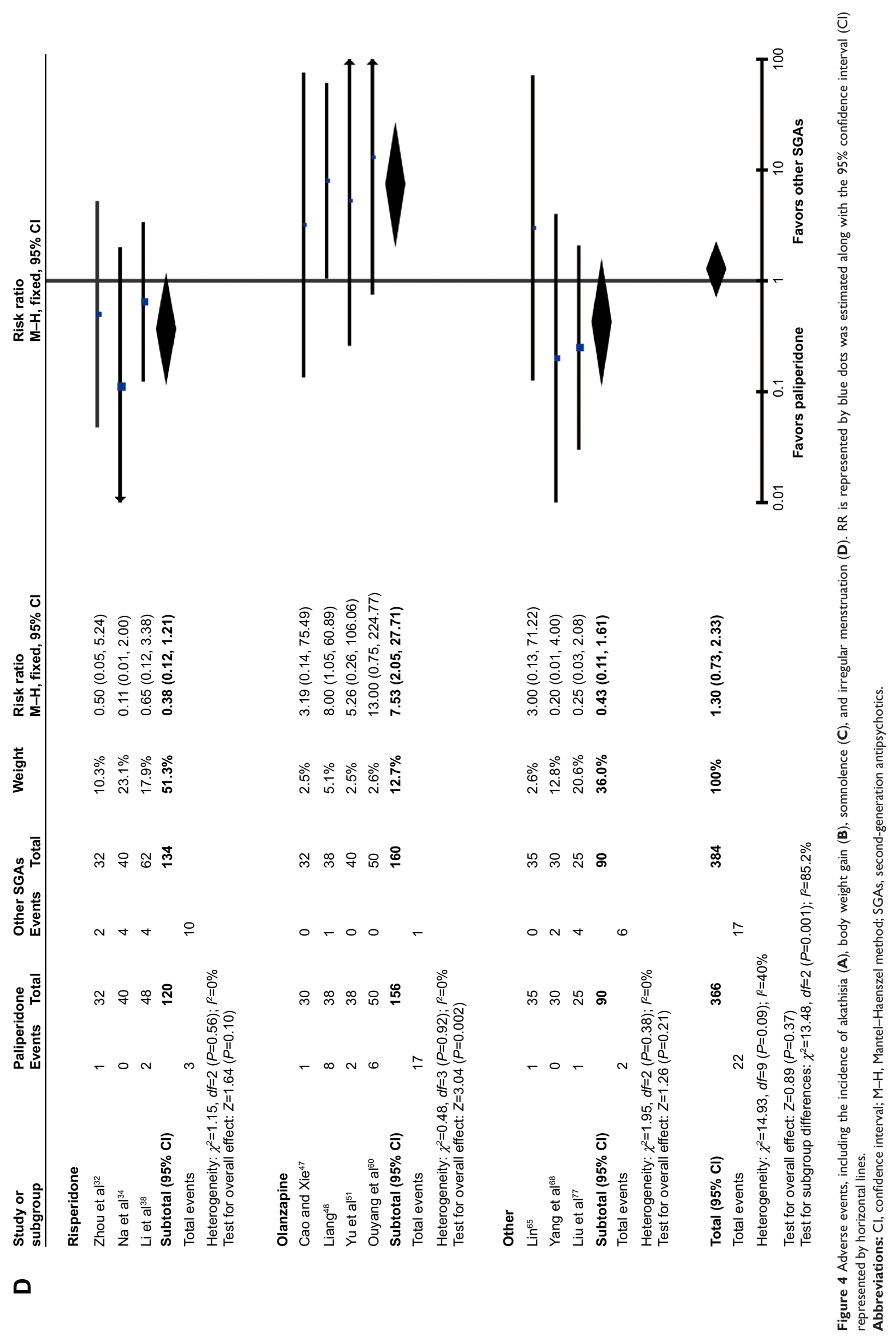


lower risk of all-cause discontinuation than risperidone ( $R R$ $0.61,95 \%$ CI $0.40-0.93, P=0.02$ ) and was comparable with olanzapine (RR $0.82,95 \%$ CI $0.53-1.27, P=0.37$ ), ziprasidone (RR 1.15, 95\% CI 0.42-3.20, $P=0.78$ ), and aripiprazole (RR $0.67,95 \%$ CI $0.12-3.74, P=0.64$ ) in this regard (Figure $5 \mathrm{~A}$ ). Further, a significant reduction in risk of all-cause discontinuation was found in the high Jadad score subgroup (RR 0.71 , 95\% CI $0.53-0.96, P=0.03)$ but not in the low Jadad score subgroup (RR 0.88, 95\% CI 0.35-2.23, $P=0.79$; Figure 5C). These results suggest that paliperidone ER is well accepted and significantly better than the pool of all other examined SGAs, as well as risperidone, specifically.

\section{Discussion}

This study investigated the efficacy and safety of paliperidone ER in Chinese patients with schizophrenia. To our knowledge, this is the first meta-analysis ever conducted in Chinese schizophrenia patients treated with paliperidone. Our results demonstrate that the response rate for paliperidone ER was significantly higher than that of ziprasidone and other pooled SGAs. Paliperidone ER significantly reduced the chance of withdrawal due to adverse events and the chance of any adverse events, compared with other pooled SGAs and risperidone. The incidence of EPS caused by paliperidone ER was comparable with other pooled SGAs and significantly lower than that of risperidone but higher than that of olanzapine. Paliperidone ER was superior to other pooled SGAs and olanzapine as far as weight gain and somnolence were concerned. Furthermore, prolactin-related adverse events caused by paliperidone ER were comparable with other pooled SGAs, but exceeded those caused by olanzapine. In addition, the acceptability of paliperidone ER, measured by odds of withdrawal for any reason, was better than that of other pooled SGAs and risperidone specifically.

In this study, the primary outcome was efficacy as measured by the response rate rather than by improvement in PANSS, Clinical Global Impression, and Brief Psychiatric Rating Scale scores. The main reason for this is that improved scores are not necessarily of any clinical significance, and the response rate can better demonstrate symptom improvement in patients and is of higher clinical value. However, 38 studies enrolled in the efficacy analysis set did not use the same criteria for treatment response. Most studies defined treatment response as an at least $30 \%$ reduction of PANSS total score. However, this definition was $25 \%$ in a few studies. This difference in measurement may have led to heterogeneity in this study.

A previously published study demonstrated that clozapine, olanzapine, risperidone, and paliperidone ER have better efficacy than quetiapine and aripiprazole, and the efficacy of paliperidone ER is not inferior to that of olanzapine and risperidone. ${ }^{16}$ These results are consistent with our study, as well as studies in other populations. ${ }^{15,16}$ However, in our meta-analysis, the response rate in patients treated with paliperidone ER was significantly higher than that in patients treated with ziprasidone, which could be inconsistent with another meta-analysis in other populations. ${ }^{16}$ Among the 50 RCTs enrolled in this study, 12 were excluded from the efficacy analysis set for lack of data on response rate. Seven of these 12 studies compared paliperidone with risperidone, which may have affected the accuracy of our analysis and increase publication bias.

Compared with other pooled SGAs, paliperidone ER reduced the chance of withdrawal due to adverse events and of any adverse events. These results are consistent with other studies. ${ }^{15}$ Also, our data suggest that the incidences of akathisia and irregular menstruation caused by paliperidone ER are comparable with other pooled SGAs, and lower than for risperidone. In addition, previous studies have demonstrated that increased plasma prolactin levels are not directly associated with symptoms such as irregular menstruation or galactorrhea, ${ }^{22,23}$ indicating that it would be more accurate to test the plasma prolactin level simultaneously. Apart from EPS and prolactinrelated adverse events, metabolic syndrome is another common adverse event caused by SGAs. Previous studies found that paliperidone ER had a lesser effect not only on metabolism ${ }^{11-13}$ but also on weight gain than risperidone, quetiapine, clozapine, and olanzapine, and its effect on sedation was comparable with that of amisulpride but better than that of other SGAs. ${ }^{15,16}$ These results are consistent with our findings.

Withdrawal for any reason was used as a measure of acceptability, and this can be affected by a number of factors, such as efficacy and safety. ${ }^{24,25}$ Consistent with other meta-analyses, ${ }^{15,16}$ our results demonstrated that paliperidone ER causes significantly lower all-cause discontinuation than other pooled SGAs, and is comparable with olanzapine, ziprasidone, and aripiprazole, suggesting good acceptability of paliperidone ER. Given that paliperidone is an active metabolite of risperidone, some physicians in People's Republic of China are not clear about the difference between these two drugs. According to our results, the efficacy of paliperidone ER and risperidone was comparable. In addition, the odds of withdrawal due to adverse events, as well as the incidence of akathisia caused by paliperidone ER, were significantly lower than that caused by risperidone. However, the acceptability 


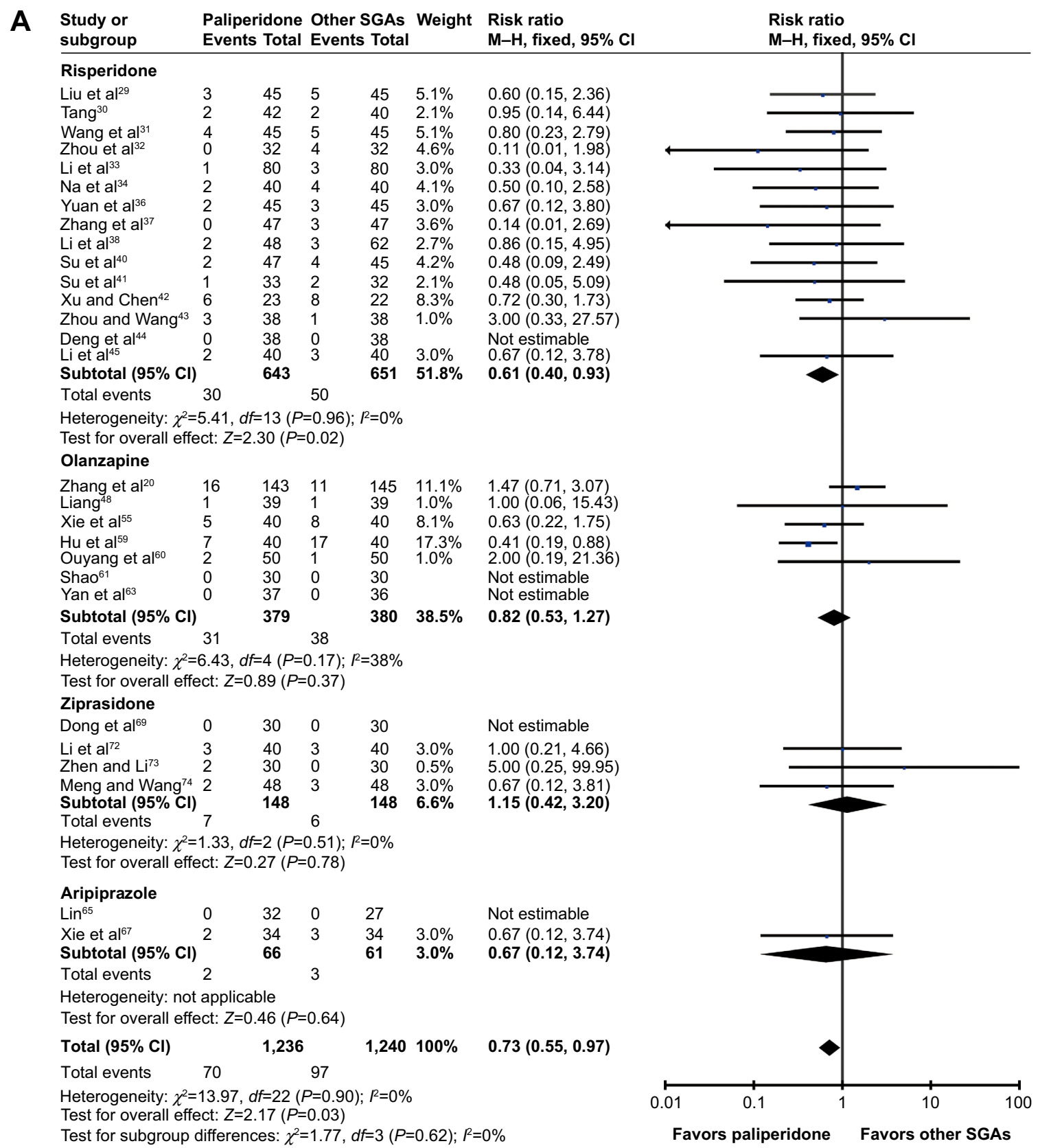

B
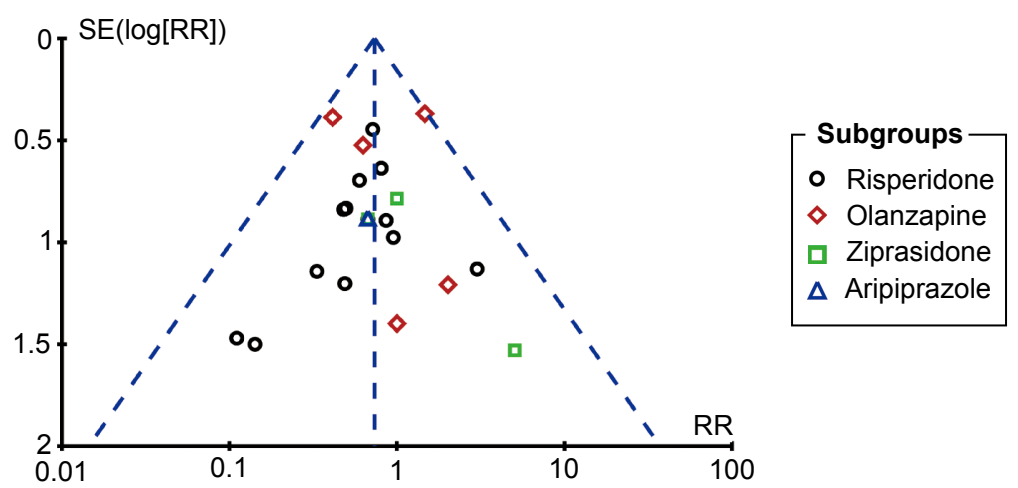

Figure 5 (Continued) 


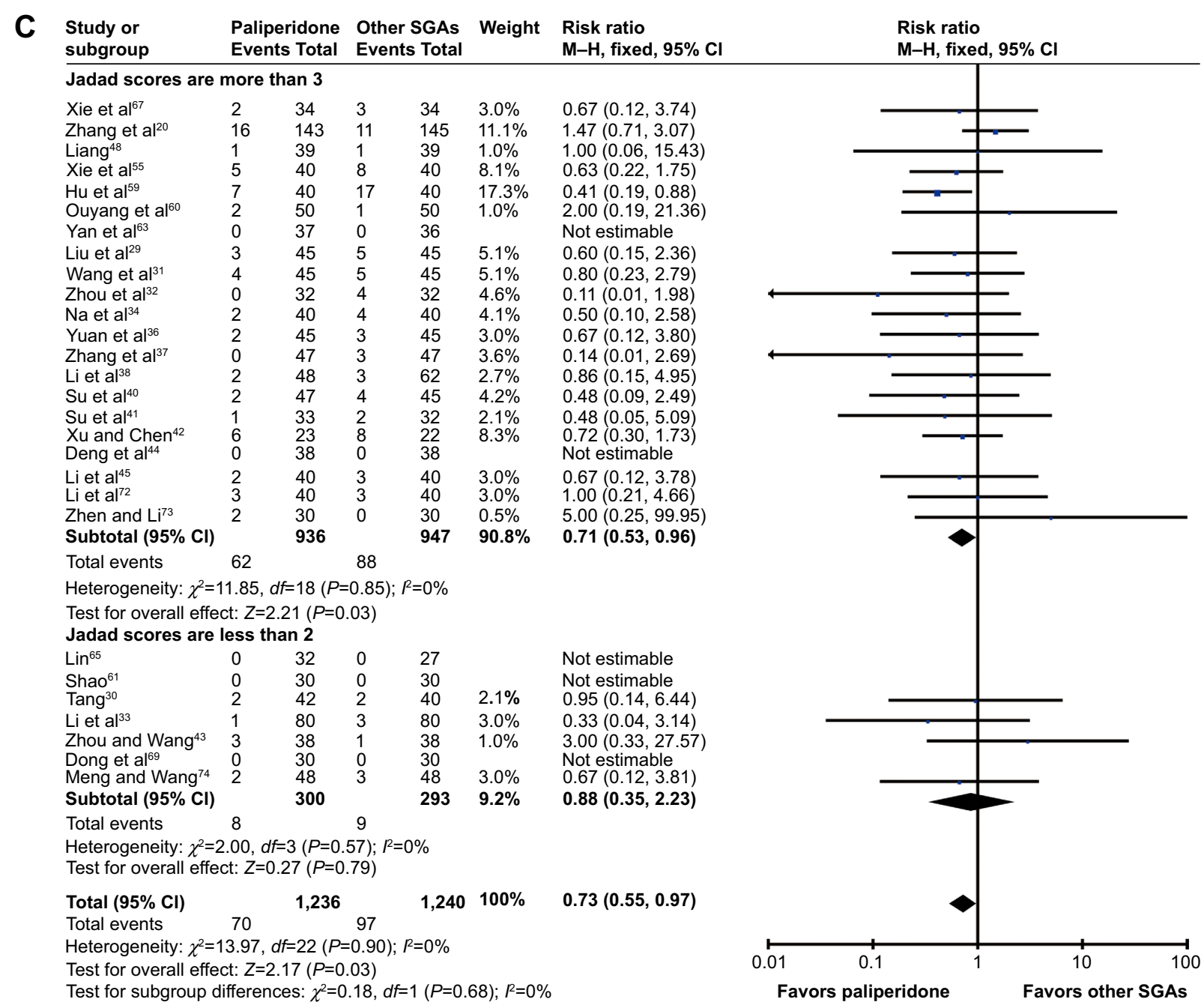

Figure 5 Withdrawal for any reason. Subgroup analysis was carried out based on treatment arms (A) and Jadad scores (C). Funnel plots were used to explore the possibility of publication bias (B). RR is represented by blue dots was estimated along with the $95 \%$ confidence interval $(\mathrm{Cl})$ represented by horizontal lines.

Abbreviations: $\mathrm{Cl}$, confidence interval; $\mathrm{M}-\mathrm{H}$, Mantel-Haenszel method; RR, risk ratio; SGAs, second-generation antipsychotics; SE, standard error.

of paliperidone ER was significantly better than that of risperidone. Some of these results might be inconsistent with other studies in other populations. ${ }^{15,16}$ Our results suggested an advantage of paliperidone ER over risperidone in Chinese patients with schizophrenia.

To increase the sensitivity of this analysis, the treatment duration in all enrolled studies had to be $4-12$ weeks so as to reduce heterogeneity between studies. To ensure the quality of the studies enrolled, all studies in Chinese had to be published in Chinese or Peking University core journals. Further, in addition to RR, we analyzed the odds ratio (OR) as another effect size at the same time. All the OR values were close to their corresponding RR values. The small difference between OR and RR values would impact neither the $P$-value nor the final conclusion.

However, this meta-analysis has several limitations. First, the number of studies enrolled in each subgroup analysis was relatively small, although funnel plots did not suggest the presence of obvious publication bias. Second, Jadad scores in half of the enrolled trials were $<3$, which may have affected the quality of the analysis. Third, several studies reported administration of anti-EPS drugs and benzodiazepines to control adverse events, which may have affected the accuracy of our analysis. Fourth, the dosage of each drug varied in the different studies, despite the recommended dose range in each study, which may have affect the heterogeneity of the studies enrolled. ${ }^{16,26}$ Further, due to limited data, our study was unable to assess improvement in social and personal functioning and some common adverse events associated with antipsychotics, such as prolonged QT interval. However, studies have demonstrated that paliperidone ER improved Personal and Social Performance scores and SF-12 Physical scores more significantly than the pool of other oral antipsychotics, indicating a role of paliperidone 
ER in improving social functioning and quality of life. ${ }^{27}$ In addition, previous studies have shown that paliperidone ER has little impact on tachycardia and metabolism, indicating good tolerance of this drug. ${ }^{14,28}$

\section{Conclusion}

Our results demonstrate that paliperidone ER is efficacious, safe, and well accepted when compared with other oral SGAs in the treatment of patients with schizophrenia, with a relatively high response rate, a low incidence of adverse events, and odds of withdrawal for any reason.

\section{Acknowledgments}

This work was supported by the National Science and Technology Major Projects for "Major New Drugs Innovation and Development" (2012ZX09303014-001, 2011ZX09302-004). The funding body had no role in the study design, data collection and analysis, decision to publish, or preparation of the manuscript. We thank Chunbo Li from the Shanghai Mental Health Center for his comments on this study.

\section{Author contributions}

All authors contributed toward data analysis, drafting and revising the paper and agree to be accountable for all aspects of the work.

\section{Disclosure}

SLC, HFL, and ZHB are employees of Janssen Research and Development. The Janssen pharmaceutical companies did not provide any financial support for this work. The authors report no other conflicts of interest.

\section{References}

1. van Os J, Kapur S. Schizophrenia. Lancet. 2009;374(9690):635-645.

2. Phillips MR, Zhang J, Shi Q, et al. Prevalence, treatment, and associated disability of mental disorders in four provinces in China during 20012005: an epidemiological survey. Lancet. 2009;373(9680):2041-2053.

3. Li Q, Xiang YT, Su YA, et al. Antipsychotic polypharmacy in schizophrenia patients in China and its association with treatment satisfaction and quality of life: findings of the third national survey on use of psychotropic medications in China. Aust NZJ Psychiatry. 2015;49(2): 129-136.

4. van Beijsterveldt LE, Geerts RJ, Leysen JE, et al. Regional brain distribution of risperidone and its active metabolite 9-hydroxy-risperidone in the rat. Psychopharmacology. 1994;114(1):53-62.

5. Gellynck E, Heyninck K, Andressen KW, et al. The serotonin 5-HT7 receptors: two decades of research. Exp Brain Res. 2013;230(4): 555-568.

6. Gray JA, Roth BL. The pipeline and future of drug development in schizophrenia. Mol Psychiatry. 2007;12(10):904-922.

7. Gyires K, Zadori ZS, Torok T, Matyus P. Alpha(2)-Adrenoceptor subtypes-mediated physiological, pharmacological actions. Neurochem Int. 2009;55(7):447-453.
8. Conley R, Gupta SK, Sathyan G. Clinical spectrum of the osmoticcontrolled release oral delivery system (OROS), an advanced oral delivery form. Curr Med Res Opin. 2006;22(10):1879-1892.

9. Vermeir M, Naessens I, Remmerie B, et al. Absorption, metabolism, and excretion of paliperidone, a new monoaminergic antagonist, in humans. Drug Metab Dispos. 2008;36(4):769-779.

10. Amatniek J, Canuso CM, Deutsch SI, et al. Safety of paliperidone extended-release in patients with schizophrenia or schizoaffective disorder and hepatic disease. Clin Schizophr Relat Psychoses. 2014;8(1): 8-20.

11. Davidson M, Emsley R, Kramer M, et al. Efficacy, safety and early response of paliperidone extended-release tablets (paliperidone ER): results of a 6-week, randomized, placebo-controlled study. Schizophr Res. 2007;93(1-3):117-130.

12. Kane J, Canas F, Kramer M, et al. Treatment of schizophrenia with paliperidone extended-release tablets: a 6-week placebo-controlled trial. Schizophr Res. 2007;90(1-3):147-161.

13. Marder SR, Kramer M, Ford L, et al. Efficacy and safety of paliperidone extended-release tablets: results of a 6-week, randomized, placebocontrolled study. Biol Psychiatry. 2007;62(12):1363-1370.

14. Rui Q, Wang Y, Liang S, et al. Relapse prevention study of paliperidone extended-release tablets in Chinese patients with schizophrenia. Prog Neuropsychopharmacol Biol Psychiatry. 2014;53:45-53.

15. Jones MP, Nicholl D, Trakas K. Efficacy and tolerability of paliperidone ER and other oral atypical antipsychotics in schizophrenia. Int J Clin Pharmacol Ther. 2010;48(6):383-399.

16. Leucht S, Cipriani A, Spineli L, et al. Comparative efficacy and tolerability of 15 antipsychotic drugs in schizophrenia: a multiple-treatments meta-analysis. Lancet. 2013;382(9896):951-962.

17. Li T, Stefansson H, Gudfinnsson E, et al. Identification of a novel neuregulin 1 at-risk haplotype in Han schizophrenia Chinese patients, but no association with the Icelandic/Scottish risk haplotype. Mol Psychiatry. 2004;9(7):698-704.

18. Li T, Li Z, Chen P, et al. Common variants in major histocompatibility complex region and TCF4 gene are significantly associated with schizophrenia in Han Chinese. Biol Psychiatry. 2010;68(7): 671-673.

19. Yu Y, Li H, Yang F, et al. [Effectiveness, tolerability and safety of flexible-dose treatment with oral extended release paliperidone in acute schizophrenia]. Shanghai Arch Psychiatry. 2010;22(6):349-353. Chinese.

20. Zhang H, Li H, Wang G, et al. [The efficacy and safety of flexible doses of paliperidone extended-release tablets in the treatment of acute schizophrenia: a randomized, double blind olanzapine-controlled study]. Chin J Psychiatry. 2009;42(3):153-157. Chinese.

21. Jadad AR, Moore RA, Carroll D, et al. Assessing the quality of reports of randomized clinical trials: is blinding necessary? Control Clin Trials. 1996;17(1):1-12.

22. Kleinberg DL, Davis JM, de Coster R, Van Baelen B, Brecher M. Prolactin levels and adverse events in patients treated with risperidone. J Clin Psychopharmacol. 1999;19(1):57-61.

23. Petty RG. Prolactin and antipsychotic medications: mechanism of action. Schizophr Res. 1999;35 Suppl:S67-S73.

24. Cipriani A, Furukawa TA, Salanti G, et al. Comparative efficacy and acceptability of 12 new-generation antidepressants: a multipletreatments meta-analysis. Lancet. 2009;373(9665):746-758.

25. Cipriani A, Barbui C, Salanti G, et al. Comparative efficacy and acceptability of antimanic drugs in acute mania: a multiple-treatments meta-analysis. Lancet. 2011;378(9799):1306-1315.

26. Rummel-Kluge C, Komossa K, Schwarz S, et al. Second-generation antipsychotic drugs and extrapyramidal side effects: a systematic review and meta-analysis of head-to-head comparisons. Schizophr Bull. 2012; 38(1):167-177.

27. Schreiner A, Hargarter L, Hitschfield K, et al. Clinical effectiveness and resource utilization of paliperidone ER for schizophrenia: Pharmacoepidemiologic International Longitudinal Antipsychotic Registry (PILAR). Curr Med Res Opin. 2014;30(7):1279-1289. 
28. Zhang H, Hao X, Wang X, et al. [An open-label study on the safety and efficacy of paliperidone extended-release in non-acute schizophrenic patients]. Chin J Psychiatry. 2012;45(1):20-24. Chinese.

29. Liu J, Yang J, Yang S, Chen Z. [The efficacy and safety of paliperidone extended-release tablets for first-episode schizophrenia]. J Practical Medicine. 2010;26(20):3775-3777. Chinese.

30. Tang J. [The efficacy and safety of paliperidone and risperidone in the treatment of patients with schizophrenia and schizophreniform psychosis: a randomized and controlled study]. Chinese $J$ Clinical Pharmacology and Therapeutics. 2010;15(9):1060-1063. Chinese.

31. Wang Y, Li W, Gu D, et al. [Efficacy of paliperidone extended-release tablets in the improvement of social functions in schizophrenics: a randomized and controlled study]. Natl Med J China. 2010;90(29) 2030-2035. Chinese.

32. Zhou P, Chen Y, Wang H, et al. [A clinical controlled study on schizophrenia treated with paliperidone-ER and risperidone]. J Clin Psychiatry. 2010;20(6):368-370. Chinese.

33. Li H, Liu Z, Wang L. [Clinical effect of paliperidone on schizophrenia]. China J Health Psychology. 2011;19(3):276-278. Chinese.

34. $\mathrm{Na} \mathrm{W}, \mathrm{Su} \mathrm{W}, \mathrm{Li}$ J, et al. [Comparison of paliperidone ER and risperidone in the treatment of schizophrenia]. Chinese J New Drugs. 2011;20(8): 708-711. Chinese.

35. Yuan G, Yu Y. [Comparison of paliperidone and risperidone in the treatment of schizophrenia negative symptoms: a randomized and controlled study]. Chinese J Clinical Pharmacology and Therapeutics. 2011;16(7):819-822. Chinese.

36. Yuan T, Qin L, Yao M, Tang J, Yue W. [The efficacy and safety of paliperidone in the treatment of 43 schizophrenia patients]. Herald Med. 2011;30(10):1288-1290. Chinese.

37. Zhang H, Liang W, Guo S. [Comparison of efficacy and safety between paliperidone and risperidone in patients with schizophrenia]. Chin J New Drugs Clin Rem. 2011;30(7):517-521. Chinese.

38. Li C, Yang G, Chen H, et al. [Efficacy, tolerability, quality of life and social functioning schizophrenia patients after treatment with oral paliperidone extended-release tablets]. Chinese J New Drugs. 2012; 21(12):1372-1376. Chinese.

39. Liu M, Liu X, Miao X, Gao X. [Effects of paliperidone extended-release tablets and risperidone on cognitive function in patients with firstepisode schizophrenia]. Chinese J New Drugs. 2012;21(4):419-422. Chinese.

40. Su W, Li J, Yang J, Guan T, Na W. [Effects of paliperidone on social functioning in schizophrenia patients: a randomized, opened, controlled trial]. Chin J New Drugs Clin Rem. 2012;31(6):345-349. Chinese.

41. Su W, Na W, Li J, Yang J, Chen H, Zhou H. [Efficacy and safety of paliperidone extended-release tablets in treatment of male patients with acute schizophrenia]. Herald Med. 2012;31(6):731-734. Chinese.

42. Xu B, Chen J. [Efficacy and safety of paliperidone in treatment of acute schizophrenia]. Chinese J Clinical Pharmacology and Therapeutics. 2012;17(2):215-219. Chinese.

43. Zhou H, Wang S. [Clinical efficacy of paliperidone in treatment of first episode schizophrenia]. J Practical Medicine. 2012;28(23):3986-3988. Chinese.

44. Deng X, Yang Y, Wang Q, Yin L. [Efficacy and safety of paliperidone extended-release and risperidone in treatment of female first-episode schizophrenia]. Chinese J Nervous Mental Disease. 2013;39(6): 355-358. Chinese.

45. Li L, Li J, Yang J, Dai X, Su W, Yu B. [A comparative study of the effects of paliperidone and risperidone on the glucose and lipid metabolism in first-episode male schizophrenia patients]. Chin Pharm J. 2013;48(8):649-651. Chinese

46. Jiang H, Jin F. [The efficacy and safety of paliperidone ER in the treatment of 30 patients with schizophrenia]. Herald Med. 2010;29(5) 631-632. Chinese.

47. Cao G, Xie G. [Observation of efficacy and safety of paliperidone sustained release tablets and domestic olanzapine in treatment of schizophrenia]. Chongqing Yixue. 2011;40(17):1695-1751. Chinese.
48. Liang Y. [A clinical comparative study on paliperidone ER in treating acute phase schizophrenia patients]. Chinese J New Drugs. 2011;20(24):2442-2445. Chinese.

49. Mao Z, Li D, Zha Z, Gu S, Jie P. [A control study of paliperidone extended-release tablets and olanzapine in the treatment of first-episode schizophrenia]. J Psychiatry. 2011;24(6):441-443. Chinese.

50. Su H, Fu W, Li D, Li Q, Chen J. [Comparative study for treatment with paliperidone release tablets and olanzapine in schizophrenia]. Chongqing Yixue. 2011;40(27):2751-2753. Chinese.

51. Yu X, Liao J, Xu J. [A controlled study of paliperidone and olanzapine in the treatment of naive schizophrenic patients]. The Journal of Clinical Psychiatry. 2011;21(4):270-271. Chinese.

52. Lv Z. [A clinical control study of paliperidone extended-release tablets and olanzapine in the treatment of female schizophrenia]. China $J$ Health Psychology. 2012;20(5):647-648. Chinese.

53. Ma Q, Li L, Lian H. [A controlled comparison study on paliperidone extended-release tablets and olanzapine in the treatment of schizophrenia]. Chinese J New Drugs. 2012;21(22):2658-2661. Chinese.

54. Qu B, Han H, Hou M. [Control study of paliperidone extended release tablets and olanzapine for schizophrenia]. J Practical Medicine. 2012;9(3):77-78. Chinese.

55. Xie Q, Feng S, Zeng H, Xun Y, Wang J. [Efficacies of paliperidone extended-release tablets and olanzapine for treating schizophrenia in children and adolescents]. Chinese J New Drugs. 2012;21(16): 1916-1919. Chinese.

56. Yang D, Chen Y. [A controlled comparison study on paliperidone extended-release tablets and olanzapine in the treatment of schizophrenia]. Journal of Clinical Psychiatry. 2012;22(4):263. Chinese.

57. Zhang X, Pei S. [Effects of paliperidone sustained release tablets and olanzapine tablets on treatment of schizophrenia]. Chinese J Health Psychology. 2012;20(2):196-197. Chinese.

58. Zhu Q, Pi J. [A comparative study of paliperidone and olanzapine in the treatment of treatment-refractory schizophrenia]. Chinese $J \mathrm{New}$ Drugs. 2012;21(6):666-669. Chinese.

59. Hu S, Yao M, Peterson BS, et al. A randomized, 12-week study of the effects of extended-release paliperidone (paliperidone ER) and olanzapine on metabolic profile, weight, insulin resistance, and beta-cell function in schizophrenic patients. Psychopharmacology. 2013;230(1):3-13.

60. Ouyang X, Yuan P, Tan L, Wan X, Zhang D. [A controlled comparison study on paliperidone extended-release tablets and olanzapine in the treatment of first-episode schizophrenia]. Guizhou Medical J. 2013;37(10):918-921. Chinese.

61. Shao G. [Clinical effect and safety of paliperidone ER on the treatment of schizophrenia]. China J Health Psychology. 2013;21(8):1133-1134. Chinese.

62. Wang H, Duan M, Ma M, Wang Q, Yan G. [A randomized controlled trial of paliperidone and olanzapine in acute schizophrenia]. Progress in Modern Biomedicine. 2013;13(4):697-700. Chinese.

63. Yan A, Liu G, Zhang G. [Paliperidone extended-release tablets and olanzapine in the treatment of schizophrenia]. China J Health Psychology. 2013;21(10):1473-1475. Chinese.

64. Guo X, Wang G, Wang Z, Wang N. [The effects of paliperidone extended-release tablets and olanzapine on the liver function of patients with schizophrenia]. Chinese J New Drugs. 2014;23(16):1941-1945. Chinese.

65. Lin W. [Efficacy of paliperidone and aripiprazole in treatment of patients with schizophrenia]. Chinese J Practical Nervous Diseases. 2012; 15(24):67-69. Chinese.

66. Liu X, Li W. [Efficacy of paliperidone in treatment of schizophrenia negative symptoms in female patients]. J Clinical Psychiatry. 2013; 23(6):371. Chinese.

67. Xie Q, Tang J, Zhou L, Zeng H. [A controlled clinical study between paliperidone and aripiprazole in adolescents with first-episode schizophrenia]. Chin J New Drugs Clin Rem. 2013;32(1):51-55. Chinese.

68. Yang G, Wu Y, Gu J, Tang L, Ji P. [Control study on paliperidone and aripiprazole in the treatment of first-episode schizophrenia]. China J Health Psychology. 2013;21(2):185-186. Chinese. 
69. Dong R, Gao X, Lu E, et al. [A comparative study of paliperidone sustained-release tablets and ziprasidone tablets for schizophrenia]. Occup and Health. 2011;27(24):2943-2944. Chinese.

70. Xu L, Xie Y, Li Y. [Efficacy and safety of paliperidone extended-release tablets in treatment of patients with schizophrenia: a randomized ziprasidone hydrochloride capsules controlled study]. The Journal of Clinical Psychiatry. 2011;21(3):174-176. Chinese.

71. He J, Tao Y, Long S, Zhou J. [Influence of paliperidone extendedrelease tablets on the social function and quality of life in patients with schizophrenia]. Chin Pharm J. 2012;47(13):1090-1093. Chinese.

72. Li L, Yang J, Dai X, Su W. [Comparative study of paliperidone and ziprasidone in the treatment of acute schizophrenia]. Herald Med. 2012;31(9):1150-1153. Chinese.

73. Zhen L, Li J. The effects of ziprasidone and paliperidone extendedrelease tablet on serum prolactin and body weight of schizophrenia. China J Health Psychology. 2012;20(12):1766-1768. Chinese.
74. Meng L, Wang S. [Comparison of efficacy and safety of paliperidone and ziprasidone in the treatment of schizophrenia]. Practical Pharmacy and Clinical Remedies. 2013;16(5):373-375. Chinese.

75. Zhang X. [Effect of paliperidone on schizophrenia and its influence on quality of life]. China J Health Psychology. 2014;22(8):1133-1135. Chinese.

76. Yu Y, Yang D, Chen Y, Zhang H, Xiong M. [Comparative study of paliperidone extended-release tablet vs quetiapine in the treatment of first episode schizophrenia]. J Clin Res. 2010;27(12):2284-2286. Chinese.

77. Liu M, Liu X, Zhang N. [A comparative study on efficacy and safety of paliperidone extended-release tablets and clozapine in treatment of resistant schizophrenia]. Chinese J New Drugs. 2012;21(14):1639-1642. Chinese.

\section{Publish your work in this journal}

Neuropsychiatric Disease and Treatment is an international, peerreviewed journal of clinical therapeutics and pharmacology focusing on concise rapid reporting of clinical or pre-clinical studies on a range of neuropsychiatric and neurological disorders. This journal is indexed on PubMed Central, the 'PsycINFO' database and CAS, and is the official journal of The International Neuropsychiatric Association (INA). The manuscript management system is completely online and includes a very quick and fair peer-review system, which is all easy to use. Visit http://www.dovepress.com/testimonials.php to read real quotes from published authors. 